\title{
FLAVONÓIDES GLICOSILADOS DAS FOLHAS E FLORES DE BAUHINIA FORFICATA (LEGUMINOSAE)
}

\author{
Moacir Geraldo Pizzolatti*, Anildo Cunha Jr., Bruno Szpoganicz e Eliandra de Sousa \\ Departamento de Química, Universidade Federal de Santa Catarina, CP 476, 88040-900 Florianópolis - SC \\ Raimundo Braz-Filho e Jan Schripsema \\ Setor de Química de Produtos Naturais, Universidade Estadual do Norte Fluminense, Av. Alberto Lamego, 2000, 28015-620 \\ Campos - RJ
}

Recebido em 5/4/02; aceito em 6/2/03

\begin{abstract}
FLAVONOIDS GLYCOSIDES FROM LEAVES AND FLOWERS OF Bauhinia forficata (LEGUMINOSAE). From the leaves of Bauhinia forficata kaempferol and four flavonoid glycosides, 3,7-di- $O$ - $\alpha$-L-rhamnopyranosylkaempferol, 3,7-di- $O$ - $\alpha$-Lrhamnopyranosylquercetin, 3- $O$-[ $\alpha$-L-rhamnopyranosyl-( $1 \rightarrow 6)-\beta$-D-glucopyranosyl]-7-O- $\alpha$-L-rhamnopyranosylkaempferol and 3$O$-[ $\alpha$-L-rhamnopyranosyl-( $1 \rightarrow 6)-\beta$-D-glucopyranosyl]-7-O- $\alpha$-L-rhamnopyranosylquercetin were obtained. From the flowers only the flavonoid 7-O- $\alpha$-L-rhamnopyranosylkaempferol was isolated. The structures were established by chemical and spectroscopic methods.
\end{abstract}

Keywords: Bauhinia forficata; Leguminosae; flavonoid glycosides.

\section{INTRODUÇÃO}

Bauhinia forficata Link (Leguminosae), uma das cerca de 300 espécies do gênero Bauhinia, apresenta-se como uma árvore de porte médio, encontrada do Rio de Janeiro ao Rio Grande do Sul, possuindo folhas uncinadas com formato semelhante a uma pata de vaca, flores brancas e frutos do tipo vagem linear ${ }^{1}$. Algumas espécies do gênero Bauhinia são utilizadas na medicina folclórica da África, Ásia e América para o tratamento de várias doenças ${ }^{2}$. A infusão das folhas de Bauhina forficata é utilizada na medicina popular brasileira como agente diurético, hipoglicemiante, tônico, depurativo, no combate à elefantíase e na redução da glicosúria ${ }^{1}$. Apesar da atividade hipoglicemiante desta espécie ter sido destacada há muito tempo ${ }^{3-5}$, poucos estudos foram realizados seja com respeito às suas atividades ou fitoquímica. O gênero Bauhinia é caracterizado pelo acúmulo de flavonóides livres e glicosilados ${ }^{6-10}$ sendo que de $B$. forficata são relatados na literatura a presença de $\beta$-sitosterol and kanferol-3,7diramnosídeo ${ }^{6}$.

O presente trabalho registra os resultados do estudo fitoquímico das folhas e flores de B. forficata, descrevendo o isolamento e a identificação de kanferol (1) e de cinco flavonóides glicosilados (26) contendo os flavonóis kanferol e quercetina como agliconas. As estruturas dos compostos foram estabelecidas por métodos químicos e espectroscópicos, principalmente técnicas uni- (1D) e bidimensionais (2D) de RMN de ${ }^{1} \mathrm{H}$ e de ${ }^{13} \mathrm{C}$.

\section{RESULTADOS E DISCUSSÃO}

O fracionamento cromatográfico em sílica gel dos extratos em AcOEt e $n$-BuOH das folhas de $B$. forficata levou ao isolamento do $\beta$-sitosterol, kanferol (1), 3,7-di- $O$ - $\alpha$-L-ramnopiranosilkanferol (3), 3,7-di-O- $\alpha$-L-ramnopiranosilquercetina (4), 3-O-[ $\beta$-D-glucopiranosíl-( $1 \rightarrow 6)-\alpha$-L-ramnopiranosil]-7- $O-\alpha$-L-ramnopiranosilkanferol $(5)$ e $3-O$-[ $\beta$-D-glucopiranosil- $(1 \rightarrow 6)-\alpha$-L-ramnopiranosil]7-O- $\alpha$-L-ramnopiranosilquercetina (6). O glicosídeo 7- $O$ - $\alpha$-L-

*e-mail: mgpizzo@qmc.ufsc.br
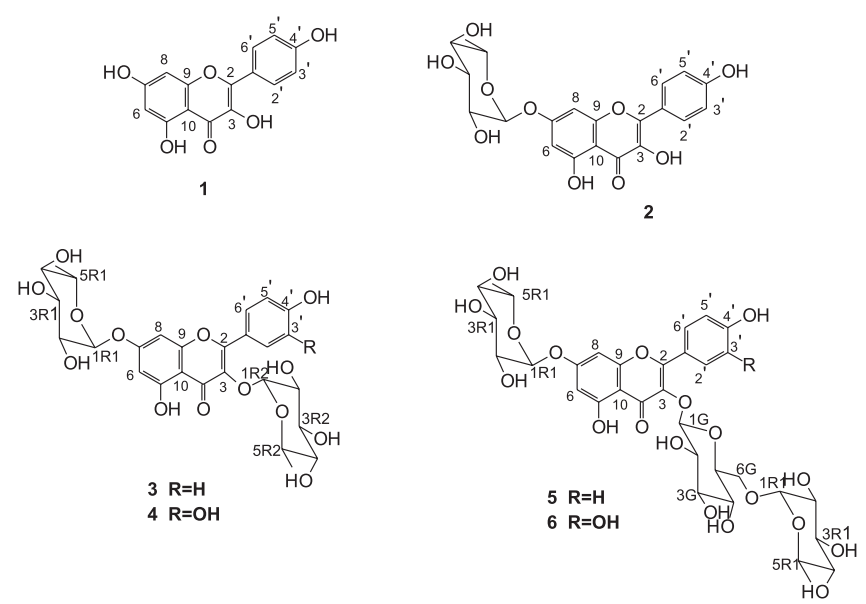

ramnopiranosilkanferol (2) foi obtido a partir do fracionamento cromatográfico do extrato em $n$ - $\mathrm{BuOH}$ das flores.

Os espectros de massas das substâncias glicosiladas 2-6 não revelaram a presença de fragmentos correspondentes aos íons moleculares, notando-se em todos estreita semelhança com o do kanferol $\left([\mathrm{M}]^{++} 286, \mathrm{C}_{15} \mathrm{H}_{10} \mathrm{O}_{6}\right)$. Nos EM de 2-6 o pico base de $\mathrm{m} / \mathrm{z}$ 286 representa a unidade aglicona com fórmula molecular $\mathrm{C}_{15} \mathrm{H}_{10} \mathrm{O}_{6}$. Nos espectros de IV observaram-se absorções de sistemas aromáti$\cos \left(v_{\max } 1600,1500 \mathrm{~cm}^{-1}\right)$, de função carbonila envolvida em ponte de hidrogênio intramolecular $\left(v_{\max } 1650 \mathrm{~cm}^{-1}\right)$ e intensas e largas absorções para grupos hidroxílicos $\left(v_{\max } 3400 \mathrm{~cm}^{-1}\right)$ sugerindo a presença de açúcares. A natureza glicosídica destas substâncias foi indicada pelo número de sinais oximetilênicos e oximetínicos observados nos espectros de $\mathrm{RMN}$ de ${ }^{1} \mathrm{H}$ e de ${ }^{13} \mathrm{C}$, e confirmada pelos espectros 2D de correlação homonuclear (COSY) e heteronuclear $\left({ }^{1} \mathrm{H}-{ }^{13} \mathrm{C}-\mathrm{HMQC}\right.$ e $\left.{ }^{1} \mathrm{H}-{ }^{13} \mathrm{C}-\mathrm{HMQC}\right)$, permitindo caracterizar as estruturas destes glicosídios como 2-6.

A unidade aglicônica dos glicosídeos $\mathbf{2}, \mathbf{3}$ e $\mathbf{5}$ foi caracterizada como 3,4',5,7-tetraidroxiflavona (kanferol) através da análise dos espectros de RMN ${ }^{1} \mathrm{H}$, reconhecendo-se os sinais de sistemas $\mathrm{AB}$, correspondente a dois átomos de hidrogênio que mantêm entre si 
relação meta e atribuídos aos hidrogênios H-6/H-8 $\left[\delta_{\mathrm{H}} 6,49 / 6,77\right.$; d; $J=2,2 \mathrm{~Hz}(\mathbf{2}) ; 6,41 / 6,81 ; \mathrm{d} ; J=1,8 \mathrm{~Hz},(\mathbf{3}) ; 6,44 / 6,79 ; \mathrm{sl},(\mathbf{5})] \mathrm{e}$ AA'BB' típico de anel B de flavonóides p-dissubstituído $\left[\delta_{\mathrm{H}} 7,78 \mathrm{e}\right.$ 6,91; d; $J=8,8 \mathrm{~Hz}(2) ; 8,07$ e 6,92; d; $J=8,8 \mathrm{~Hz}$ (3); 8,08 e 6,86; d; $J=8,8(\mathbf{5})]$. A análise por CCF dos produtos da hidrólise ácida com solução alcóolica a $10 \%$ de $\mathrm{HCl}$ dos glicosídeos $\mathbf{2}$ e $\mathbf{3}$ revelou a presença de kanferol e ramnose e a hidrólise de $\mathbf{5}$ resultou em kanferol, ramnose e glicose.

A fórmula molecular $\mathrm{C}_{21} \mathrm{H}_{20} \mathrm{O}_{10}$ do glicosídeo 2 foi deduzida pela comparação dos espectros de RMN ${ }^{13} \mathrm{C}\left\{{ }^{1} \mathrm{H}\right\}$ (1D) e 2D HMQC, permitindo reconhecer os sinais correspondentes aos átomos de carbono não hidrogenados (nove carbonos $\mathrm{sp}^{2}$ ), metínicos (quatro sinais representando seis carbonos $\mathrm{sp}^{2}$ e cinco $\mathrm{sp}^{3}$ oxigenados, entre eles um anomérico em $\delta_{\mathrm{C}} 98,94$ correlacionado com o sinal em $\delta_{\mathrm{H}} 5,54$, singleto largo, no espectro HMQC) e metílico (grupo metila da ramnose em $\delta_{\mathrm{C}}$ 17,94 correlacionado com o sinal em $\delta_{\mathrm{H}} 1,12$, dubleto com $J=6,2 \mathrm{~Hz}$, no HMQC). A presença de uma unidade ramnopiranose foi confirmada pela análise dos demais sinais dos espectros de $\mathrm{RMN}^{1} \mathrm{H}$ e $\mathrm{RMN}^{13} \mathrm{C}$
(Tabela 1). A localização desta unidade estrutural no átomo de oxigênio ligado ao carbono C-7 da aglicona baseou-se nos seguintes dados espectrais: a) o deslocamento químico do hidrogênio anomérico em $\delta_{\mathrm{H}} 5,54(\mathrm{~d}, J=1,8 \mathrm{~Hz}$, hidrogênio $\mathrm{H}-1$ da unidade ramnopiranose em posição equatorial) revelou-se compatível com a localização do açúcar na posição 7-OH, que foi confirmada pela interação heteronuclear deste hidrogênio $\left({ }^{3} J_{\mathrm{CH}}\right)$ com o C-7 $\left(\delta_{\mathrm{C}} 161,42\right)$ revelada pelo espectro $2 \mathrm{D} \mathrm{HMBC}$ (Tabela 2); b) o sinal em $\delta_{\mathrm{H}} 12,45$ no espectro de $\mathrm{RMN}^{1} \mathrm{H}$ indicou a presença de HO-5, posição de absorção típica de hidroxila quelatogênica em anel de seis membros; c) o sinal de hidroxila em $\delta_{\mathrm{H}}$ 10,10 foi atribuído ao grupo hidroxila 4' $-\mathrm{OH}$ pelo acoplamento heteronuclear a longa distância ( $\left.{ }^{3} J_{\mathrm{CH}}\right)$ com os carbonos CH-3' e CH-5' $\left(\delta_{\mathrm{C}} 115,48\right)$ observado no espectro HMBC (Tabela 2$)$, onde também se observa correlação entre o hidrogênio 9,44 ppm (HO-3) e o carbono C-2 $\left(\delta_{C} 147,50\right)$. Assim, a atribuição dos deslocamentos químicos dos átomos de carbono (Tabela 1) e hidrogênio, baseada nas análises detalhadas dos espectros 1D e 2D de RMN, e a comparação com os dados da literatura ${ }^{12}$ permitiram definir a estrutura deste glicosídio como

Tabela 1. Dados de RMN ${ }^{1} \mathrm{H}$ e ${ }^{13} \mathrm{C}$ do kanferol (1) e dos glicosídeos 2, 3 e 5, registrado em DMSO- $d_{6}$ usando aparelho que opera a $600 \mathrm{MHz}$ para hidrogênio e $150 \mathrm{MHz}$ para carbono-13. Deslocamentos químicos em $\delta\left(\delta_{\mathrm{H}}\right.$ e $\left.\delta_{\mathrm{C}}\right)$ e constantes de acoplamento (J, entre parênteses) em $\mathrm{Hz}^{*}$

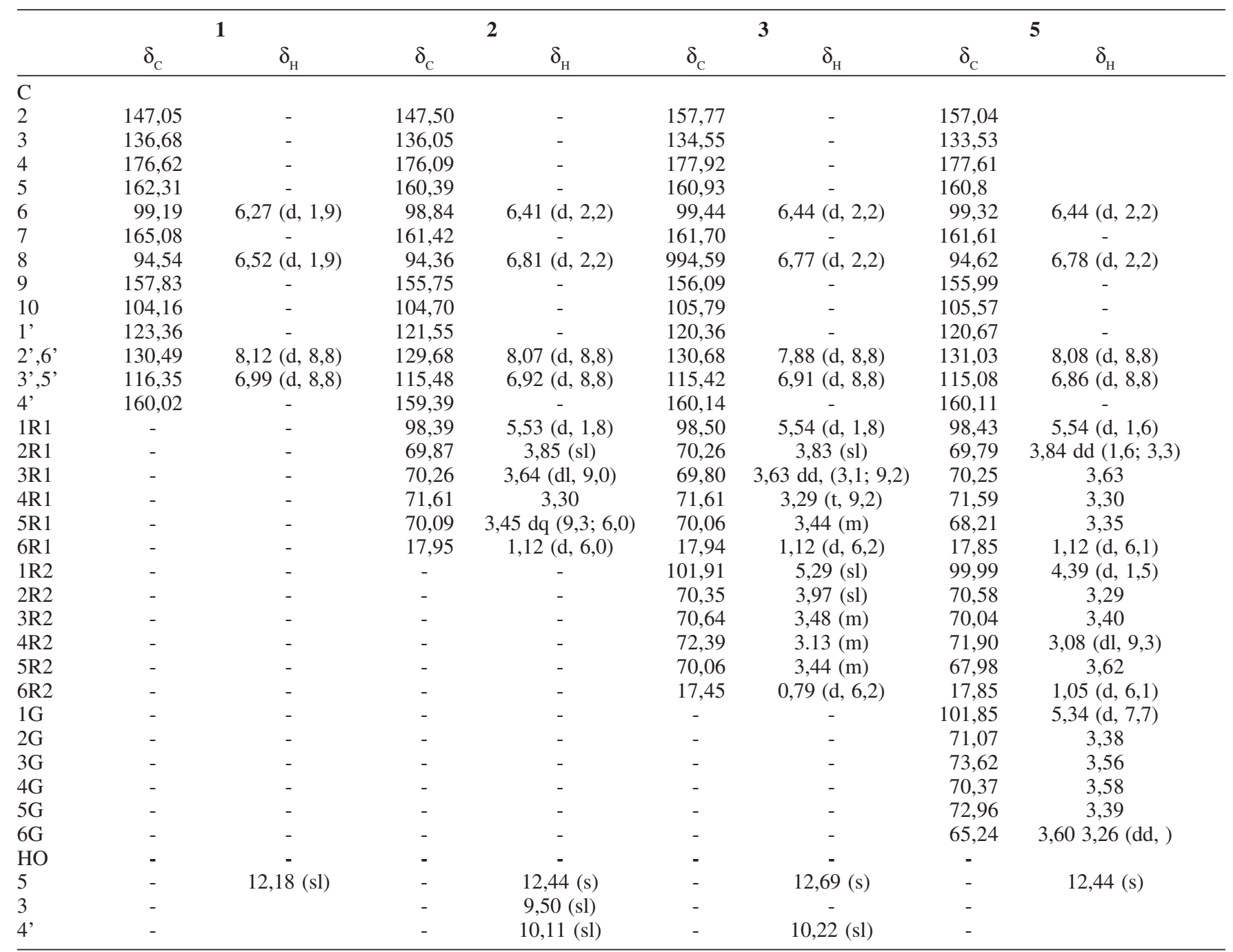

*Os sinais dos átomos de carbono quaternários, metínicos e metílicos foram reconhecidos pela análise comparativa dos espectros DEPT135. Os espectros 2D de correlação heteronuclear ${ }^{1} \mathrm{H}-{ }^{13} \mathrm{C}$ - HMQC e ${ }^{1} \mathrm{H}-{ }^{13} \mathrm{C}-\mathrm{HMBC}$ foram também usados na atribuição dos deslocamentos químicos. Os deslocamentos químicos de átomos de hidrogênio sem indicação de multiplicidade indicam sinais envolvidos em superposição e os valores descritos representam aproximações deduzidas pelos espectros 2D HMQC e HMBC. 
Tabela 2. Dados de interação heteronuclear spin-spin a longa distância $\left({ }^{2} \mathrm{~J}_{\mathrm{CH}} \mathrm{e}^{3} \mathrm{~J}_{\mathrm{CH}}\right)$ de átomos de carbono e hidrogênio obtidos dos espectros HMBC de 2 e $\mathbf{5}^{*}$

\begin{tabular}{|c|c|c|c|c|c|c|}
\hline \multicolumn{4}{|c|}{2} & \multicolumn{3}{|c|}{5} \\
\hline $\mathbf{C}$ & $\delta_{\mathrm{C}}$ & ${ }^{2} \mathrm{~J}_{\mathrm{CH}}$ & ${ }^{3} \mathbf{J}_{\mathrm{CH}}$ & $\delta_{\mathrm{C}}$ & ${ }^{2} \mathrm{~J}_{\mathrm{CH}}$ & ${ }^{3} \mathbf{J}_{\mathrm{CH}}$ \\
\hline 2 & 147,50 & - & HO-3; 2H-2',6' & 157,05 & - & $2 \mathrm{H}-2^{\prime}, 6^{\prime}$ \\
\hline 3 & & & & 133,55 & - & $\mathrm{H}-1 \mathrm{G}$ \\
\hline 5 & 160,39 & HO-5; H-6 & - & 160,84 & H-6 & \\
\hline 6 & 98,84 & - & $\mathrm{HO}-5 ; \mathrm{H}-8$ & 99,32 & - & $\mathrm{H}-8$ \\
\hline 7 & 161,42 & H-6; H-8 & 1-HR1 & 161,61 & H-6; H-8 & H-1R1 \\
\hline 8 & 94,36 & - & H-6 & 94,62 & - & H-6 \\
\hline 9 & 155,75 & $\mathrm{H}-8$ & - & 155,99 & $\mathrm{H}-8$ & - \\
\hline 10 & 104,70 & - & HO-5; H-6; H-8 & 105,57 & - & H-6; H-8 \\
\hline 1 ' & 121,55 & & 2H-3",5' & 120,67 & & $2 \mathrm{H}-3^{\prime}, 5$ \\
\hline $3^{\prime}, 5^{\prime}$ & 115,48 & & HO-4' & 115,08 & & \\
\hline $4^{\prime}$ & 159,39 & HO-4'; 2H-3',5' & $2 \mathrm{H}-2^{\prime}, 6^{\prime}$ & 160,11 & $2 \mathrm{H}-3^{\prime}, 5^{\prime}$ & $2 \mathrm{H}-2^{\prime}, 6^{\prime}$ \\
\hline 1R1 & 98,39 & & & & & \\
\hline 2R1 & 69,87 & H-1R1; H-3R1 & $\mathrm{H}-4 \mathrm{R} 1$ & 69,79 & H-1R1 & \\
\hline $3 \mathrm{R} 1$ & 70,26 & $\mathrm{H}-2 \mathrm{R} 1$ & H-1R1 & 70,25 & H-2R1; H-4R1 & H-1R1 \\
\hline $4 \mathrm{R} 1$ & 71,61 & H-5R 1 & $3 \mathrm{H}-6 \mathrm{R} 1$ & 71,59 & H-3R1 & $3 \mathrm{H}-6 \mathrm{R} 1$ \\
\hline $5 \mathrm{R} 1$ & 70,09 & H-4R1; 3H-6R1 & H-1R1 & 68,21 & $3 \mathrm{H}-6 \mathrm{R} 1$ & H-3R1 \\
\hline 1R2 & - & - & - & 99,99 & & \\
\hline $2 \mathrm{R} 2$ & - & - & - & 70,58 & $\mathrm{H}-2 \mathrm{R} 2$ & \\
\hline $3 \mathrm{R} 2$ & - & - & - & 70,04 & & \\
\hline $4 \mathrm{R} 2$ & - & - & - & 71,90 & & $3 \mathrm{H}-6 \mathrm{R} 2$ \\
\hline $5 \mathrm{R} 2$ & - & - & - & 67,98 & $3 \mathrm{H}-6 \mathrm{R} 2$ & H-1R2 \\
\hline $6 \mathrm{R} 2$ & - & - & - & 17,85 & & H-4R2 \\
\hline $1 \mathrm{G}$ & - & - & - & 101,85 & & $\mathrm{H}-3 \mathrm{G}$ \\
\hline $2 \mathrm{G}$ & - & - & - & 71,07 & & \\
\hline $3 \mathrm{G}$ & - & - & - & 73,62 & & $\mathrm{H}-5 \mathrm{G}$ \\
\hline $4 G$ & - & - & - & 70,37 & & \\
\hline $5 \mathrm{G}$ & - & - & - & 72,95 & & H-3G \\
\hline $6 \mathrm{G}$ & - & - & - & 65,24 & & $\mathrm{H}-1 \mathrm{G} ; \mathrm{H}-4 \mathrm{G}$ \\
\hline
\end{tabular}

* Os sinais dos átomos de carbono quaternários, metínicos e metílico foram reconhecidos pela análise comparativa dos espectros DEPT135. Os espectros 2D de correlação heteronuclear HMQC foram também usados na atribuição dos deslocamentos químicos. Os deslocamentos químicos dos átomos de hidrogênio $\left(\mathrm{d}_{\mathrm{H}}\right)$ encontram-se descritos na Tabela 1.

7-O- $\alpha$-L-ramnopiranosilkanferol (2), anteriormente isolado de Platanus acerifolia ${ }^{11}$.

Após a caraterização e a atribuição dos deslocamentos químicos dos átomos de hidrogênio $\left(\delta_{\mathrm{H}}\right)$ e carbono $\left(\delta_{\mathrm{C}}\right)$ de 3 (Tabela 1 ), anteriormente isolado nesta espécie ${ }^{6}$, verificou-se a existência de sinais adicionais nos espectros de $\mathrm{RMN}^{1} \mathrm{H}$ e ${ }^{13} \mathrm{C}$, que permitiram postular a presença de 3,7-di- $O$ - $\alpha$-L-ramnopiranosílquercetina (4), presente em menor percentagem. Os deslocamentos químicos dos sinais de átomos de hidrogênio em $\delta_{\mathrm{H}} 6,43(\mathrm{~d}, J=2,1 \mathrm{~Hz}, \mathrm{H}-6), 6,77$ (d, $J=2,1$ Hz, H-8), 6,74 (d, $J=1,7 \mathrm{~Hz}, \mathrm{H}-2^{\prime}$ ), 6,87 (d, $\left.J=8,3 \mathrm{~Hz}, \mathrm{H}-5^{\prime}\right)$ e $7,28,\left(\mathrm{dd}, J=8,3\right.$ e $1,7 \mathrm{~Hz}, \mathrm{H}-6$ ') e de carbono em $\delta_{\mathrm{C}} 120,58$ (C-1'), 115,72 (CH-2'), 145,23 (C-3'), 148,61 (C-4'), 114,91 (CH-5') e 121,21 (CH-6') observados nos espectros de $\mathrm{RMN}^{1} \mathrm{H}$ e $\mathrm{RMN}^{13} \mathrm{C}$, respectivamente, permitiram deduzir a estrutura 4. A análise por $\mathrm{CCF}$ também revelou a presença de um componente minoritário com $\mathrm{Rf}$ menor que o Rf de $\mathbf{3}$, justificando-se a maior polaridade de $\mathbf{4}$ pela presença de uma hidroxila no átomo de carbono C-3'.

O composto 5 foi caracterizado como um kanferol triglicosilado. As três unidades glicosídicas foram identificadas como duas ramnoses e uma glicose com base nos dados espectrais de $\mathrm{RMN}{ }^{1} \mathrm{H}$ e RMN ${ }^{13} \mathrm{C}$. A presença de uma unidade $\alpha$-L ramnopiranosídica ligada ao oxigênio do carbono C-7 foi deduzida pelos sinais em $\delta_{\mathrm{H}} 5,54$ (singleto largo, $\mathrm{H}-1 \mathrm{R} 1$, hidrogênio $\mathrm{H}-1$ da ramnose em posição equatorial) e $\delta_{\mathrm{C}} 161,61$ atribuído ao C-7, como observado em 2 e 3. A confirmação veio do espectro HMBC (Tabela 2) através do acoplamento heteronuclear $\left({ }^{3} J_{\mathrm{CH}}\right)$ entre o hidrogênio anomérico $\mathrm{H}$ $1 \mathrm{R} 1\left(\delta_{\mathrm{H}} 5,54\right)$ e o carbono C-7 $\left(\delta_{\mathrm{C}} 161,61\right)$. A caracterização e a localização da unidade diglicosídica $\alpha$-L-ramnopiranosil- $(1 \rightarrow 6)-\beta$ D-glicopiranosil no átomo de oxigênio do carbono C-3 está fundamentada nas seguintes observações: a) o deslocamento químico do átomo de carbono $\mathrm{C}-2\left(\delta_{\mathrm{C}} 157,05\right)$ revelado pelo espectro de $\mathrm{RMN}{ }^{13} \mathrm{C}$ sugeriu a presença de glicosídeo em C-3, como observado em 3 (Tabela 1); b) a correlação a longa distância entre o hidrogênio anomérico H-1G $\left(\delta_{\mathrm{H}} 5,34, \mathrm{~d}, J=7,7 \mathrm{~Hz}\right.$, hidrogênio da glicose em posição axial) e o carbono $\mathrm{C}-3\left(\delta_{\mathrm{C}} 133,53,{ }^{3} \mathrm{~J}_{\mathrm{CH}}\right)$, observada no espectro HMBC (Tabela 2), permitiu definir a ligação 3-O- $\beta$-D-glicopiranosila; c) o deslocamento químico do átomo de carbono metilênico $\mathrm{CH}_{2}-6 \mathrm{G}\left(\delta_{\mathrm{C}}\right.$ $65,25)$ permitiu a localização da unidade ramnose restante neste átomo de carbono, já que o sinal de grupo hidroximetilênico livre de uma unidade glicopiranosila aparece em torno de $\delta_{\mathrm{C}} 62^{12} ; \mathrm{d}$ ) o espectro HMBC (Tabela 2) revelou a interação heteronuclear do hidrogênio anomérico H-1R2 $\left(\delta_{\mathrm{H}} 4,39\right.$, singleto largo de hidrogênio em posição equatorial) da ramnose com o carbono $\mathrm{CH}_{2}-6 \mathrm{G}\left(\delta_{\mathrm{C}} 65,25\right) \mathrm{da}$ glucose. Assim, esta estrutura pode ser caracterizada como sendo 3$O$-[ $\alpha$-L-ramnopiranosil- $(1 \rightarrow 6)-\beta$-D-glucopiranosil]-7- $O-\alpha$-Lramnopiranosilkanferol (5) e os deslocamentos químicos de RMN de ${ }^{13} \mathrm{C}$ indubitavelmente atribuídos. Embora esta estrutura já tenha sido descrita anteriormente como constituinte de Lens esculenta ${ }^{13}$, não foram encontrados dados de RMN de ${ }^{13} \mathrm{C}$ descritos.

$\mathrm{O}$ espectro de $\mathrm{RMN}^{1} \mathrm{H}$ de $\mathbf{5}$ mostrou a presença de sinais adicionais correspondentes a um componente minoritário contendo a quercetina como aglicona. Esta dedução baseou-se na presença dos sinais em $\delta_{\mathrm{H}} 6,42$ (d, $\left.J=2,2 \mathrm{~Hz}, \mathrm{H}-6\right), 6,75$ (d, $\left.J=2,2 \mathrm{~Hz}, \mathrm{H}-8\right), 7,57$ (d, $J=2,2 \mathrm{~Hz}, \mathrm{H}-2^{\prime}$ ), 6,82 (d, $J=8,4 \mathrm{~Hz}, \mathrm{H}-5$ ') e 7,67 (dd, $J=8,5$ e 
2,1 Hz, H-6') observados no espectro de $\mathrm{RMN}{ }^{1} \mathrm{H}$ e na análise por $\mathrm{CCF}$, que revelou a presença de componente em menor percentagem com menor Rf. Estes resultados permitem postular a presença de 3$O$-[ $\alpha$-L-ramnopiranosil- $(1 \rightarrow 6)-\beta$-D-glucopiranosil]-7- $O-\alpha-\mathrm{L}$ ramnopiranosilquercetina $(\mathbf{6})$.

A identificação do kanferol e do glicosídeo 3 foi realizada por comparação direta dos dados de ressonância com a literatura ${ }^{14,15}$.

Neste trabalho foram caracterizados quatro flavonóides glicosilados 2, 4, 5 e 6 e o kanferol que estão sendo descritos pela primeira vez como constituintes de $B$. forficata e são apresentados os dados de ressonância ainda não descritos para o glicosídeo $\mathbf{5}$.

\section{PARTE EXPERIMENTAL}

\section{Procedimento geral}

Os PF foram determinados em um aparelho da Microquímica APF-301 sem correções. Os espectros IV foram registrados em pastilhas de $\mathrm{KBr}$ em um espectrofotômetro Perkin Elmer FT 16PC. Os espectros de $\mathrm{RMN}{ }^{1} \mathrm{H}$ e ${ }^{13} \mathrm{C}$ foram obtidos em um espectrômetro Bruker DRX $600\left({ }^{1} \mathrm{H}\right.$ : $600 \mathrm{MHz} ;{ }^{13} \mathrm{C}$ : $\left.150 \mathrm{MHz}\right)$ a $300{ }^{\circ} \mathrm{K}$; os programas padrões de pulso com gradiente de campo INVIETGSSI e INV4GSLPLRND da Bruker foram usados para obtenção dos espectros 2D HMQC e HMBC, respectivamente; os experimentos foram ajustados para constantes de acoplamento de 140 e 7,7 Hz, respectivamente, usando-se probe multinuclear de $5 \mathrm{~mm}$ ("triple resonance inverse probehead"), tetrametilsilano como padrão interno e DMSO- $d_{6}$ como solvente. Os espectros de massa obtidos por impacto de elétrons a $70 \mathrm{eV}$ foram registrados em um espectrômetro Shimadzu CG-MS-QP-2000.

\section{Material vegetal}

As folhas e flores de Bauhinia forficata foram coletadas na cidade de Orleans (Santa Catarina) em Novembro de 1998. A planta foi identificada pelo Prof. D. de B. Falkenberg do Departamento de Botânica da Universidade Federal de Santa Catarina, Florianópolis, SC, Brasil, onde uma exsicata da espécie foi depositada sob o número FLOR-31271.

\section{Preparação do extrato bruto e frações}

As folhas $(960 \mathrm{~g})$ e flores $(200 \mathrm{~g})$ secas e trituradas foram extraídas separadamente por maceração exaustiva com EtOH: $\mathrm{H}_{2} \mathrm{O}(7: 3)$ à temperatura ambiente. Os extratos hidroalcoolicos foram evaporados sob pressão reduzida a $50{ }^{\circ} \mathrm{C}$ até $1 / 4$ de seus volumes iniciais e mantidos em repouso por dois dias a $4{ }^{\circ} \mathrm{C}$. Em seguida, estas soluções e os filtrados foram submetidos a sucessivos particionamentos com $n$-hexano, $\mathrm{CH}_{2} \mathrm{Cl}_{2}$, AcOEt e $n$-ButOH. O solvente de cada fase orgânica foi evaporado para a obtenção dos respectivos extratos das folhas e flores.

\section{Isolamento dos flavonóides das folhas e flores}

$\mathrm{O}$ extrato em AcOEt das folhas $(16,13 \mathrm{~g})$ foi submetido à cromatografia em coluna (CC) de sílica gel usando como eluente misturas de polaridade crescente de $n$-hexano/AcOEt $/ \mathrm{MeOH}$, obtendo-se frações de $100 \mathrm{~mL}$. A fração 9 foi recristalizada em metanol e forneceu $10 \mathrm{mg}$ de $\beta$-sitosterol. As frações 70-84 e 94-98 forneceram $\mathbf{1}$ (20 mg) e $\mathbf{3}$ (83 mg), respectivamente, após recristalizações com AcOEt. O extrato em $n$-BuOH das folhas $(11,80 \mathrm{~g})$ foi submetido ao mesmo procedimento descrito acima, recolhendo-se 25 fra- ções de $100 \mathrm{~mL}$; as frações 9-16 forneceram 5 (25 mg) após recristalização em AcOEt. O extrato em $n$ - $\mathrm{BuOH}$ das flores $(6 \mathrm{~g})$ foi também fracionado em CC de sílica, usando-se o mesmo procedimento aplicado para as frações acima descritas. Foram coletadas 54 frações de $100 \mathrm{ml}$. As frações 27 a 33, reunidas com base na análise por $\mathrm{CCF}$, após sucessivas recristalizações com AcOEt forneceram 2 $(8,5 \mathrm{mg})$.

\section{Hidrólise ácida}

Cada um dos glicosídeos 2, 3 e $\mathbf{5}$ foram dissolvidos em EtOH$10 \% \mathrm{HCl}$ e refluxados por $2 \mathrm{~h}$. A mistura resultante foi diluída com água e extraída com EtOAc. Os açúcares foram identificados na fase aquosa por CCF ( $\mathrm{Si} \mathrm{Gel} \mathrm{tamponada} \mathrm{com} \mathrm{acetato} \mathrm{de} \mathrm{sódio} \mathrm{0,02M;}$ $\mathrm{CHCl}_{3}-\mathrm{MeOH} 3: 2$; revelador: anizaldeído-sulfúrico) e comparação com amostras autênticas.

Kanferol (1): sólido amorfo amarelo. PF: $278-280{ }^{\circ} \mathrm{C}$. IV $v_{\text {máx }}$ $\mathrm{cm}^{-1}: 3384,1660,1610,1514,1494$. EMIE $\mathrm{m} / \mathrm{z}$ (int. rel) $286(100$ $\left.[\mathrm{M}]^{+}\right)$. $\mathrm{RMN}^{1} \mathrm{H} \mathrm{e}^{13} \mathrm{C}\left(\mathrm{CDCl}_{3}\right)$ : Tabela 1.

7-O- $\alpha$-L-ramnopiranosilkanferol (2): sólido amorfo amarelo. PF: 208,4-209,5 ${ }^{\circ} \mathrm{C}$. IV $v_{\text {máx }} \mathrm{cm}^{-1}: 3322,1658,1590,1554,1496$. EMIE $m / z$ (int. rel) $286\left(100[\mathrm{M}]^{+}\right)$. RMN ${ }^{1} \mathrm{He} \mathrm{e}^{13} \mathrm{C}\left(\mathrm{DMSO}-d_{6}\right)$ : Tabelas $1 \mathrm{e}$ 2.

3,7-di-O- $\alpha$-L-ramnopiranosilkanferol (3): sólido amorfo amarelo. PF: $198,5-201,3{ }^{\circ} \mathrm{C}$. IV $v_{\text {máx }} \mathrm{cm}^{-1}: 3340,1650,1592$. EMIE $m / z$ (int. rel) $286\left(100[\mathrm{M}]^{++}\right)$. $\mathrm{RMN}^{1} \mathrm{H} \mathrm{e}^{13} \mathrm{C}$ (DMSO- $\left.d_{6}\right)$ : Tabela 1.

3-O-[ $\alpha$-L-ramnopiranosil-( $1 \rightarrow 6)-\beta-D$-glucopiranosíl]-7-O- $\alpha-L$ ramnopiranosilkanferol (5): sólido amorfo amarelo. PF: $184-190{ }^{\circ} \mathrm{C}$. IV $v_{\text {máx }} \mathrm{cm}^{-1}: 3376,1656,1592,1546,1488$. EMIE m/z (int. rel) 286 $\left(100[\mathrm{M}]^{+}\right) \cdot \mathrm{RMN}^{1} \mathrm{He} \mathrm{e}^{13} \mathrm{C}\left(\mathrm{DMSO}-d_{6}\right)$ : Tabelas 1 e 2.

\section{AGRADECIMENTOS}

Os autores agradecem ao Prof. D. de B. Falkenberg do Departamento de Botânica da Universidade Federal de Santa Catarina pela identificação da planta, ao Departamento de Bioquímica da Universidade Federal do Rio de Janeiro pelas facilidades oferecidas para a utilização do aparelho Bruker de RMN e ao CNPq, CAPES e FAPERJ pelos auxílios e bolsas concedidos.

\section{REFERÊNCIAS}

1. Martins, R. E.; Castro, D. M.; Castellani, D. C.; Dias, J. E.; Plantas Medicinais, Ed. UFV : Viçosa, 1998, p. 155.

2. Achenbach, H.; Stocker, M.; Constenla, M. A.; Phytochemistry 1988, 27, 1835 .

3. Juliane, C.; Rev. Med. Pharm. Chim. Phys. 1931, 2, 165.

4. Juliane, C.; Rev. Sudam Endocri. Immol. Quimiot. 1931, 14, 326.

5. Costa, O. A.; Rev. Flora Medicinal 1945, 9, 15.

6. Silva, K. L.; Biavatti, M. W.; Leite, S. L.; Yunes, R. A.; Delle Monache, F.; Cechinel, V.; Z. Naturforsch. 2000, 55, 478.

7. Yadava, R. N.; Tripathi, P.; Fitoterapia 2000, 71, 88.

8. Kuo, Y.; Yeh, M.; Huang, S.; Phytochemistry 1998, 49, 2529.

9. Viana, E. P.; Santa-Rosa, R. S.; Almeida, S. S. M. S.; Santos, L. S.; Fitoterapia 1999, 70, 111.

10. Salatino, A.; Salatino, M. L. F.; Giannasi, D. E.; Biochem. Syst. Ecol. 2000, 28,545 .

11. Kaouadji, M.; Phytochemistry 1990, 29, 2295.

12. Agrawal, P. K.; Phytochemistry 1992, 31, 3307.

13. EI-Negoumy, S. I.; EI-Sayed, N. H.; Mabry, T. J.; Rev. Latinoamer. Quim. 1987, $18,88$.

14. Agrawal, P. K.; Bansal, M. C. Em Carbon-13 NMR of Flavonoids; Agrawal, P. K, ed.; Elsevier: Amsterdam,1989.

15. Aragão, P. C. de A.; de Toledo, J. B.; Morais, A. A.; Braz-Filho, R.; Quim. Nova 1990, 13, 254. 